\title{
Spacetime as a Feynman diagram: the connection formulation
}

\author{
Michael P. Reisenberger \\ Theoretical Physics Group, Blackett Laboratory, \\ Imperial College, London, SW7 2BZ, UK. \\ Center for Gravitational Physics and Geometry, The Pennsylvania \\ State University, 104 Davey Lab, University Park, PA16802-6300, USA \\ m.reisenberger@ic.ac.uk \\ Carlo Rovelli \\ Physics Department, University of Pittsburgh, \\ Pittsburgh, PA 15260, USA. \\ Centre de Physique Théorique, CNRS Luminy, 13288 Marseille, France \\ rovelli@cpt.univ-mrs.fr \\ May 29, 2018
}

\begin{abstract}
Spin foam models are the path integral counterparts to loop quantized canonical theories. In the last few years several spin foam models of gravity have been proposed, most of which live on finite simplicial lattice spacetime. The lattice truncates the presumably infinite set of gravitational degrees of freedom down to a finite set. Models that can accomodate an infinite set of degrees of freedom and that are independent of any background simplicial structure, or indeed any $a$ priori spacetime topology, can be obtained from the lattice models by summing them over all lattice spacetimes. Here we show that this sum can be realized as the sum over Feynman diagrams of a quantum
\end{abstract}


field theory living on a suitable group manifold, with each Feynman diagram defining a particular lattice spacetime. We give an explicit formula for the action of the field theory corresponding to any given spin foam model in a wide class which includes several gravity models. Such a field theory was recently found for a particular gravity model [1]. Our work generalizes this result as well as Boulatov's and Ooguri's models of three and four dimensional topological field theories, and ultimately the old matrix models of two dimensional systems with dynamical topology. A first version of our result has appeared in a companion paper [2]: here we present a new and more detailed derivation based on the connection formulation of the spin foam models.

\section{Introduction}

The spin foam formalism [3, 4, 5, 6, 7, 8, 9] has emerged in the last few years as an elegant synthesis of several approaches to quantum gravity and diffeomorphism invariant theories more generally. It can be viewed as a "path integral" formulation corresponding to the canonical loop quantization framework 10, 11, 12, 13, 14] and also as an extension of the simplicial framework for topological field theories [15, 16, 17, 18], which allows more general, non-topological, field theories to be represented.

Spin foams are coloured two dimensional complexes consisting of two dimensional faces (of arbitrary topology), joined on edges of valence $\geq 3$. Faces are coloured with non-trivial irreducible representations of a "gauge group" G] while edges carry "intertwiners" - $G$ invariant tensors in the product representation formed by the representations on the incident faces. In a spin foam model the spin foams are regarded as histories of the physical system and assigned quantum amplitudes. In models of gravity a spin foam defines a discrete spacetime geometry [4, 15, 19]; Spin foam models of gravity incorporate the discreteness of the geometry of space first uncovered [20, 21, 22, 23, 24] in loop quantized canonical theory into a spacetime sum over histories formalism naturally suited to a 4-diffeo invariant theory. This is very appealing because the discreteness, which is not "put in by hand" but arises naturally

\footnotetext{
1 We will see that $G$ corresponds to the local gauge group or fibre bundle structure group commonly refered to as the "gauge group" in discussions of Yang-Mills theory, as opposed to the group of all gauge transformations of the fields in spacetime.
} 
in this framework, ₹ promises to remove the ultraviolet divergences found in perturbative theories of quantum gravity and matter fields.

A number of spin foam models of Euclidean [5, 6, 26, 27, 28] and Lorentzian [29, 30, 31] quantum gravity in four spacetime dimensions have been proposed. Three dimensional general relativity [15, 3, 25] as well as four dimensional BF theory [17, 18, 4] can also be given a spin foam formulation. Even hypercubic Yang-Mills theory can be expressed in this framework. In fact any lattice model in which a connection with a compact gauge group forms the boundary data can be translated into a spin foam model [6].

Of the spin foam models of gravity referred to above all but [5] and [30] are simplicial lattice models. A shortcoming of such models is that their predictions depend on the simplicial complex chosen to represent spacetime. Topological field theories, such as three dimensional general relativity or BF theory, can be formulated on a triangulated manifold in such a way as to be independent of the particular triangulation of the manifold chosen, essentially because such theories have only a finite number of degrees of freedom associated to global topological features of the manifold. Four dimensional gravity models on the other hand are expected to have infinitely many degrees of freedom in any given spacetime topology, so any finite triangulation (in which each simplex carries a finite set of degrees of freedom as in the proposed models) necessarily truncates and thus misrepresents the gravitational field.

Some sort of continuum limit must be taken. One approach is to sum over triangulations of the spacetime manifold. This seems very difficult to

\footnotetext{
${ }^{2}$ In using the spin foam formalism, or the canonical loop quantization formalism one is making an ansatz, so it is possible that the discreteness is not real because the whole framework is not the one used by Nature. In lattice spin foam models there is another possibility, namely that an infinite renormalization of the bare theory that is necessary to go to the continuum limit wipes out the discreteness of the geometry in this limit. Finally it should be noted that in a spin foam model of Lorentzian $2+1$ general relativity developed by Freidel 25] geometrical observables do not have an entirely discrete spectrum but there is a non-zero minimal length. This is a result of the non-compactness of the three dimensional Lorentz group $S O(2,1)$ and might be a general feature of Lorentzian models.

3 These models are not Euclidean field theories in the ordinary sense because essentially the exponential of $i$ times the action is used to weight histories. We call these models of Euclidean quantum gravity because they are proposals for quantizations of classical general relativity with metric signature ++++ .
} 
do in three or more dimensions since it is difficult to identify the topology of a given simplicial complex. A technically easier, but also more ambitous approach would be to sum over all simplicial manifoldsf and thus sum over both triangulations and spacetime topology. It is an old dream of gravity theorists to make topology dynamical, thus removing yet another element of a priori background structure. It would be interesting to sum even models formulated on a continous spacetime manifold, such as those found in [5, 8] and 30], over spacetime topologies.

In the present paper we will consider another, even wider, summation. Simplicial spin foams live on the 2-skeleton of the cellular complex dual to the simplicial complex. In other words, the spin foam faces consist of dual 2-cells, and the spin foam edges of dual 1-cells. The dual 2-skeleton, the two dimensional complex formed by these cells (together with the dual 0cells), thus is spacetime as far as spin foams are concerned. We therefore propose to sum spin foam models of four spacetime dimensional gravity over all "admissible" 2-complexes - 2-complexes that preserve some simple local features of dual 2-skeletons of triangulated 4-manifolds. Specifically we will require of admissible 2-complexes that their $0-, 1-$, and 2- cells are topologically points, line segments, and disks respectively. Furthermore we require that the combinatorial structure at each 0 -cell is that of a dual 2-skeleton to a triangulated 4-manifold. Thus each 0-cell has incident on it five endpoints of 1-cells and ten corners of 2-cells with each incident 2-cell corner bounded by two of the incident 1-cells. This corresponds to the fact that a 4 -simplex (dual to a 0-cell) has in its boundary five 3-simplices (dual to 1-cells) and ten 2-simplices (dual to 2-cells). (See Fig. 1). In a sufficiently small neighborhood of a 0-cell the 2-complex is indistinguishable from a dual 2-skeleton of a 4-d simplicial complex. Note however that we do not forbid 1-cells and 2-cells from being incident several times on the same 0-cell, so some fairly strange 2-complexes are included in the sum.

Our main result is that this summation of a spin foam model over 2complex spacetimes may be realized as the sum over Feynman diagrams in a perturbation expansion of a quantum field theory: The spin foam model defines a (somewhat unusual) quantum field theory on the Cartesian product

${ }^{4}$ Simplicial manifolds are simplicial complexes that are also manifolds. To be a manifold a complex must satisfy several local requirements the most non-trivial of which is that the boundary of the union of the simplicies incident on a point is a sphere. 

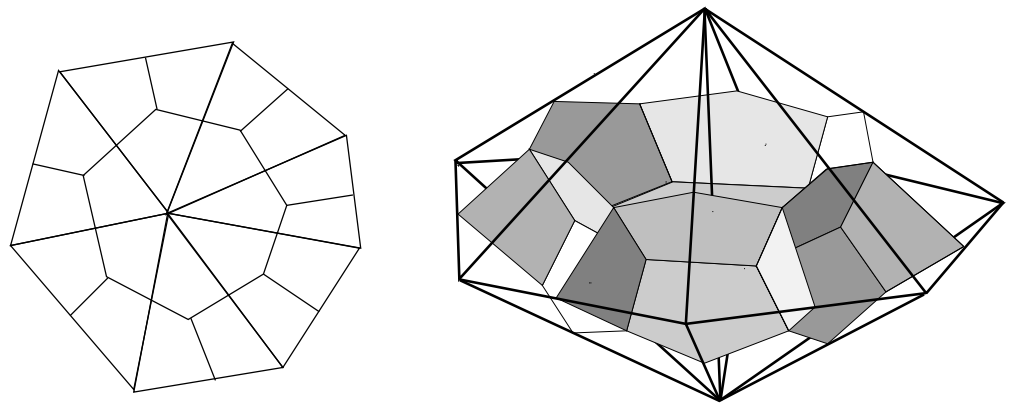

Figure 1: This figure illustrates simplicial complexes and their dual skeletons in two and three dimensions, as an aid to the reader contemplating the the four dimensional simplicial complexes and their dual 2-skeletons that appear in our discussions. On the left a complex of seven triangles (2-simplices) along with its dual 1-skeleton is shown. On the right a complex of six 3simplices (sharing a common 1-simplex) and its dual 2-skeleton is shown, with the dual 2-cells shaded in. In both cases the dual skeleton is cut off at the boundary of the simplicial complex. 
of four copies of the gauge group $G$ the Feynman diagrams of which are precisely the admissible 2-complexes, with the amplitude of each given, modulo symmetry factors, by the spin foam sum on that 2-complex. (Comparing with a familiar QFT such as scalar $\lambda \phi^{4}$ theory in Minkowski space we see that the spacetime 2-complex plays the role of the graph of a Feynman diagram, while the spin foams living on the the 2-complex play the roles of the values of the momenta on the edges of a Feynman diagram, which are integrated over to obtain the amplitude of the diagram). We propose to adopt the perturbation series of the field theory, with its symmetry factors, as the definition of the spin foam model summed over 2-complex spacetimes.

To make things more concrete let's consider the amplitude for given boundary data on the boundary of a spacetime region. This amplitude is the sum of the amplitudes of the histories that match the boundary data. In a spin foam model on a fixed 2-complex $J$ representing spacetime the boundary is a graph $\Gamma=\partial J$ and the sum runs over all spin foams living on $J$ matching the boundary data - irreducible representations on the edges of $\Gamma$ and intertwiners on the nodes. Now we wish to sum the amplitude obtained by summing over spin foams also over all $J$ bounded by the same, fixed, $\Gamma$, which is to be considered part of the boundary data. In the field theory picture this sum over 2-complexes is the Feynman diagram expansion of the expectation value of an observable that encodes the boundary data. The field theory formulation provides us with formal functional integral expressions for this expectation value, and any other quantitiy of interest, which are often easier to manipulate than the original sums. Moreover, a regular-

\footnotetext{
${ }^{5}$ This amplitude can be thought of as the quantum probability amplitude of the boundary data in the Hartle-Hawking vacuum. Alternatively, if the boundary is divided into past and future parts then the amplitude can be interpreted as the transition amplitude from past to future data.

${ }^{6}$ In the sum we are contemplating $\Gamma$ need not be the boundary of $J$ in quite the usual sense. We allow the possibility that parts of $\Gamma$ are glued together instead of to $J$. The situation is similar to that of encountered with shrink wrapped foods in which part of the plastic covering is stuck to itself rather than the food item. Returning to a more theoretical setting, this peculiar feature of the boundaries we admit is physically quite natural. If we divide $\Gamma$ into a future half $\Gamma_{+}$and a past half $\Gamma_{-}$and interpret the amplitude of the boundary data as the transition amplitude from the past to the future boundary data, then there is no reason to exclude complexes representing "bubble" time evolution in which parts of $\Gamma_{+}$and $\Gamma_{-}$coincide, and no reason to exclude such complexes from a sum over complexes interpolating between $\Gamma_{+}$and $\Gamma_{-}$.
} 
ization scheme that renders well defined the functional integral would also provide a definition of the values of the sums. We shall return to this point.

Our work generalizes the results of Ooguri [17] and De Pietri et. al. [1] who obtained field theory formulations for two particular models (summed over spacetime 2-complexes), namely $S U(2)$ BF theory [32] and the BarrettCrane model [27] of Euclidean quantum gravity. Many of the underlying ideas go back to the work of Boulatov [33], on three dimensional gravity, and to matrix models of two dimensional theories [34].

The remainder of the paper is organized as follows. In 82 we define precisely the class of spin foam models under consideration and then we present and verify our main result: an explicit formula for the action of the field theory that defines the sum over 2-complexes of any given spin foam model in this class. The Turaev-Ooguri-Crane-Yetter model of BF theory is discussed as an example. Throughout this section spin foam models are formulated as lattice gauge theories on a special type of lattice. In $\S 3$ we show how these spin foam models, and the field theory generating the sum over spacetime 2-complexes, are represented in terms spin foams. The issue of divergences, regularization and renormalization is also briefly touched on. In the last section we give an argument suggesting that the field theory is actually finite for regulated spin foam models (which is not at all obvious from the perspective of a sum over spacetime 2-complexes) and outline how the theory can be extended to accomodate more general spin foam models.

\section{Field theory formulation of spin foam mod- els with dynamical topology}

\subsection{Local spin foam models in a lattice connection for- mulation}

In the present section we obtain, explicitly, a field theory defining the sum over 2-complex spacetimes for a wide class of "local" spin foam models living on the dual 2-skeletons of triangulated 4-manifolds, or more generally on admissible 2-complexes. This class includes all Euclidean simplicial four dimensional gravity models except that of Iwasaki [28].

Instead of working directly with spin foam sums we will use the connec- 

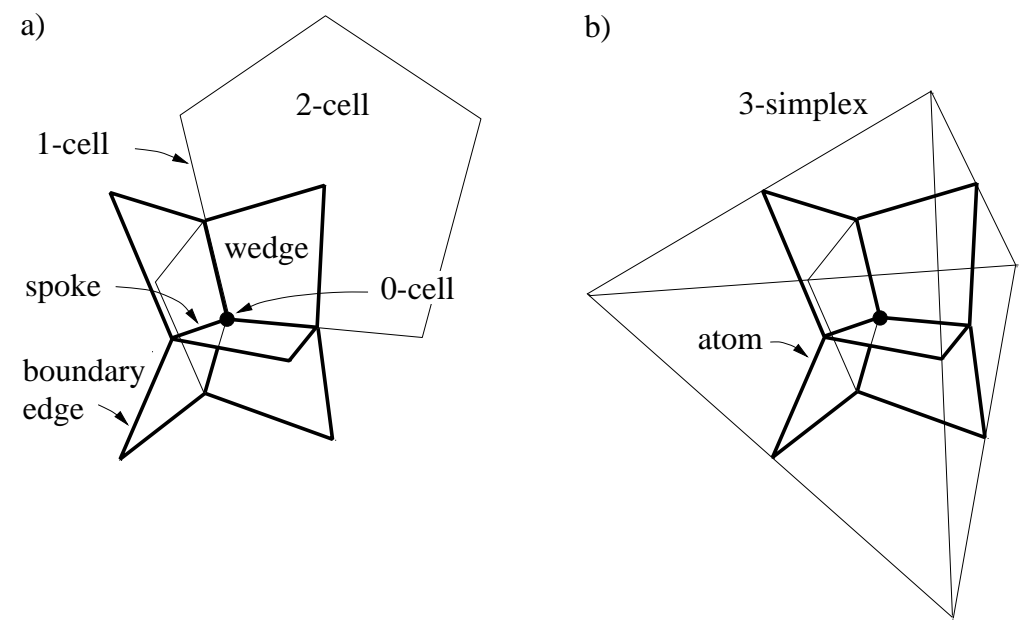

Figure 2: Atoms and their relation to simplices are illustrated in three dimensions. Panel a) shows an atom and one complete dual 2-cell of which the atom has a wedge. Panel b) shows the 3 -simplex inside which the atom would live were it part of a dual 2-complex of a three dimensional simplicial complex. The "four dimensional" atoms that we deal with in the text differs from that shown in that it is identical to the portion inside a 4-simplex of the 2 -skeleton dual to a four dimensional simplicial complex. This means that the atom has five one dimensional cells and ten wedges, as opposed to the four one dimensional cells and six wedges shown here.

tion formulation of the spin foam models because this provides the easiest route to our result. In $\S$ 爮 we will show how the result looks in the spin foam formulation and indicate how it may be obtianed within that framework. In the connection formulation a history consists of a connection specified by elements of $G$ defining parallel transport along "boundary edges" which run from the center of a 2-cell of $J$ to the center of one of its bounding 1-cells.[] As illustrated in Fig. 22 a) these edges cut $J$ into disjoint, topologically identical

\footnotetext{
${ }^{7}$ Where the centers of these cells are placed does not really matter. In fact the parallel transporters could simply be associated with pairs consisting of a 2-cell and a 1-cell in its boundary.
} 
pieces which we shall call "atoms", because they can be viewed as the fundamental building blocks from which any admissible 2-complex can be built. Each atom contains one 0-cell, five one dimensional cells called "spokes" (portions of 1-cells of $J$ ), and ten two dimensional cells called "wedges" (portions of 2-cells of $J$ ). It is bounded by a graph made of boundary edges that is homeomorphic to the 1 -skeleton $\Gamma_{5}$ of a 4 -simplex, having five 4 -valent nodes connected in all possible ways by chains consisting of two boundary edges. The 4-valent nodes are the centers of incident 1-cells while the 2-valent nodes in the chains are the centers of incident 2-cells. (Henceforth when we speak of the nodes of the atomic boundaries we shall mean only the 4 -valent nodes unless the 2-valent nodes are explicitly included). When $J$ is the dual 2skeleton of a four dimensional simplicial complex the boundary edges are precisely the edges where the boundaries of the 4-simplices cut the dual 2skeleton, and the atoms that they cut $J$ into are the portions of $J$ inside each 4 -simplex. Atoms are in this sense the dual 2-skeletons of 4-simplices. See Fig. 目 b).

The models we shall consider have compact $G$ and they are local. Each is defined by a "vertex function" $V$, a gauge invariant function of a connection on $\Gamma_{5}$. This function evaluated on the connection on the boundary of an atom gives the quantum mechanical amplitude for that connection. The amplitude for the whole connection on all of $J$ is then the product of the amplitudes for all of the atoms.

If we number the 4 -valent nodes of $\Gamma_{5}$ from 1 to 5 and let the indices $i, j, k, \ldots$ range over these numbers then we may indicate the oriented edge of $\Gamma_{5}$ from node $i$ to node $j$ by $l_{i j}$ and the half edge from $i$ to the center of $l_{i j}$ (which corresponds to a boundary edge in the boundary of an atom) by $e_{i}{ }^{j}$. $V$ is then a function of the parallel transporters $g_{i j}=h_{i}{ }^{j}\left[h_{j}{ }^{i}\right]^{-1}$ along the $l_{i j}$, where $h_{i}{ }^{j} \in G$ is the parallel transporter along $e_{i}{ }^{j}$. In the model the amplitude for one atom, $x$, is thus $V\left(h_{x i}{ }^{j}\left[h_{x j}{ }^{i}\right]^{-1}\right)$ where $h_{x i}{ }^{j}$ is the parallel transporter along the boundary edge mapped to $e_{i}{ }^{j}$, and the amplitude for the connection on the whole spacetime is

$$
w=\prod_{x \in \text { atoms of } J} V\left(h_{x i}{ }^{j}\left[h_{x j}{ }^{i}\right]^{-1}\right) .
$$

(Since each atom contains one 0 -cell the product can also be viewed as a product over 0-cells of $J) .{ }^{0}$

\footnotetext{
${ }^{8}$ A footnote on vector and matrix notation: In (1) $h_{x i}^{j}$ indicates the whole matrix of
} 
If the model is a simplicial approximation to a spacetime manifold field theory, as all the gravity models aim to be, then $V$ is an approximation to $\exp (i$ [Effective action in 4-simplex] $)$ in terms of the connection on the boundary of the atom in the 4-simplex. The atomic boundary is a graph in the boundary of the simplex so the connection on it is a discrete approximation to the continuum connection on the boundary of the 4-simplex.

As is proper for a local theory regions of spacetime (composed of whole atoms) communicate only via boundary data - the connection on the boundary. A model in this connection formalism is nothing other than a lattice gauge theory defined on an unusual lattice in order to bring boundary data and locality to the fore. For this reason this class of models was called "local lattice gauge theory" in [6].

\subsection{The main result: the field theory that generates the sum over topologies}

We can now introduce the field theory that generates the sum over admissible 2-complexes of the model defined by the vertex function $V$. It is a real scalar field theory on $G^{4}$, the Cartesian product of four copies of the gauge group manifold, determined by the action

$$
I[\psi]=I_{0}[\psi]-\lambda \mathcal{V}[\psi]
$$

where

$$
I_{0}[\psi]=\frac{1}{2 \cdot 4 !} \int_{G^{4}} d^{4} h \psi^{2}\left(h_{1}, h_{2}, h_{3}, h_{4}\right)
$$

and

$$
\mathcal{V}[\psi]=\frac{1}{5 !} \int_{G^{20}} d^{20} h V\left(h_{i}{ }^{j}\left[h_{j}{ }^{i}\right]^{-1}\right) \psi\left(h_{1}{ }^{i}\right) \psi\left(h_{2}{ }^{i}\right) \psi\left(h_{3}{ }^{i}\right) \psi\left(h_{4}{ }^{i}\right) \psi\left(h_{5}{ }^{i}\right) .
$$

group elements $\left[h_{x i}^{j}\right]_{1 \leq i<j \leq 5}$ in a sort of abstract index notation. It should be clear from the context when such expressions denote a whole matrix or vector or just one element. On other occasions we will use boldface letters to denote matrices or vectors, so the matrix of $h_{x i}{ }^{j}$ S would be written as $\mathbf{h}_{x}$ and the second row of this matrix can be written as $\mathbf{h}_{x 2}$. Again information that is clear from the context will be left out, with $\mathbf{h}$ in one context refering to all $h \mathrm{~s}$ in the whole 2-complex while in another only to the four $h$ s associated with a given node on an atomic boundary. Finally we shall use the Einstein summation convention on gauge group tensor indices, so any repeated index of this type is summed over. 
$I_{0}$ is the kinetic term. It is quadratic, but it contains no derivatives. $\lambda \mathcal{V}$ is a 5 th order, non-local interaction term, with $\lambda$ the coupling constant in which we will expand to get the perturbation series. The scalar field $\psi\left(h_{1}, h_{2}, h_{3}, h_{4}\right)$ is required to be symmetric in its four arguments.

A wavefunction $\theta$ of the connection on boundary $\Gamma$ is represented by the observable 9

$$
\Theta[\psi]=\frac{1}{\operatorname{sym}(\Gamma)} \int d \mathbf{h}_{\Gamma} \theta\left(\mathbf{h}_{\Gamma}\right) \prod_{a \in \text { nodes of } \Gamma} \psi\left(h_{a}^{b}\right) .
$$

The arguments $h_{a}{ }^{b}$ of $\psi$ are the parallel transporters along the boundary edges $e_{a}{ }^{b}$ from the node $a$ toward the four neighboring nodes (indexed by $b) ; \mathbf{h}_{\Gamma}$ represents the whole connection on $\Gamma$, i.e. the vector of all the parallel transporters along boundary edges in $\Gamma$; and $\operatorname{sym}(\Gamma)$ is the number of symmetries of $\Gamma$, i.e. the number of mappings of the set of nodes of $\Gamma$ to itself that preserve the adjacency matrix. (Since the identity mapping is a $\operatorname{symmetry} \operatorname{sym}(\Gamma) \geq 1)$.

The main result of the present paper is the following:

\section{Theorem}

The formal perturbation series of the expectation value of $\Theta^{*}$,

$$
\int \mathcal{D} \psi e^{-I_{0}[\psi]+\lambda \mathcal{V}[\psi]} \Theta^{*}[\psi]
$$

in powers of $\lambda$ is

$$
\sum_{J \in \mathbf{A}_{\Gamma}} \frac{\lambda^{n(J)}}{\operatorname{sym}(J)} \int d \mathbf{h} \theta^{*}\left(\mathbf{h}_{\Gamma}\right) w(\mathbf{h}),
$$

the sum over the set $\mathbf{A}_{\Gamma}$ of admissible 2-complexes $J$ bounded by $\Gamma$ of the overlap of the state $\theta$ with the Hartle-Hawking vacuum of the spin foam model on $J . n(J)$ is the number 0-cells in $J$ and $\operatorname{sym}(J)$ is the number of symmetries of $J$, i.e. of mappings of $J$ to itself that preserve the combinatorial structure of $J$. $\mathcal{D} \mathcal{D} \psi$ is normalized so that $\int \mathcal{D} \psi e^{-I_{0}[\psi]}=1$.

\footnotetext{
9 The observable, mentioned in the introduction, that represents a particular boundary connection $\mathbf{h}_{\Gamma}$ is obtained by taking $\theta$ to be a delta distribution on the gauge equivalence class of $\mathbf{h}_{\Gamma}$.

10 The identity mapping is included in this set so $\operatorname{sym}(J)$ is at least 1.
} 
Proof: The proof is similar to the derivation the Feynman diagram expansion in local field theories in Minkowski space. The order $\lambda^{n}$ term in (6) is

$$
\frac{\lambda^{n}}{n !} \int \mathcal{D} \psi e^{-I_{0}[\psi]} \mathcal{V}^{n}[\psi] \Theta^{*}[\psi]
$$

The functional integral is Gaussian. It's value is zero if the number of factors of $\psi$ in $\mathcal{V}^{n} \Theta^{*}$ is odd. If the number is even the value of the integral is a sum of terms associated with each possible partition of the factors of $\psi$ in $\mathcal{V}^{n} \Theta^{*}$ into pairs. Here we imagine that $\mathcal{V}^{n} \Theta^{*}$ has been written as $n$ factors of $\mathcal{V}$ followed by $\Theta^{*}$ and that the $\mathcal{V}_{\text {s }}$ and $\Theta^{*}$ have been written explicitly as the integrals (4) and (5) with the factors of $\psi$ in the integrals appearing in some definite order. The pairings being summed over are the distinct pairings of the elements in the ordered sequence of $\psi$ s appearing in this explicit expression for $\mathcal{V}^{n} \Theta^{*}$. The term corresponding to a given pairing is obtained by replacing each pair $\psi(\mathbf{h}) \psi\left(\mathbf{h}^{\prime}\right)$ by the corresponding propagator

$$
\begin{aligned}
\left\langle\psi(\mathbf{h}) \psi\left(\mathbf{h}^{\prime}\right)\right\rangle_{0} & \equiv \int \mathcal{D} \psi e^{-I_{0}[\psi]} \psi(\mathbf{h}) \psi\left(\mathbf{h}^{\prime}\right) \\
& =-\int \mathcal{D} \psi \psi(\mathbf{h}) \sum_{\sigma \in S_{4}} \frac{\delta}{\delta \psi\left(\sigma\left[\mathbf{h}^{\prime}\right]\right)} e^{-I_{0}[\psi]} \\
& =\sum_{\sigma \in S_{4}} \int \mathcal{D} \psi \frac{\delta \psi(\mathbf{h})}{\delta \psi\left(\sigma\left[\mathbf{h}^{\prime}\right]\right)} e^{-I_{0}[\psi]} \\
& =\sum_{\sigma \in S_{4}} \delta\left(h_{1}, h_{\sigma(1)}^{\prime} \delta\left(h_{2}, h_{\sigma(2)}^{\prime}\right) \delta\left(h_{3}, h_{\sigma(3)}^{\prime}\right) \delta\left(h_{4}, h_{\sigma(4)}^{\prime}\right) .\right.
\end{aligned}
$$

Here $\mathbf{h}$ and $\mathbf{h}^{\prime}$ are the sequences of four group element arguments of $\psi$, and $\sigma\left[\mathbf{h}^{\prime}\right]=\left[h_{\sigma(1)}^{\prime}, \ldots, h_{\sigma(4)}^{\prime}\right]$ is $\mathbf{h}^{\prime}$ reordered according to the permutation $\sigma$. Note that the symmetrization had to be introduced in (10) because the integral runs over symmetrized $\psi$ only. Thus the integration by parts is justified only for the symmetrized derivative.⿴囗十

$\mathcal{V}^{n} \Theta^{*}$ is an integral over the connections on the boundaries of $n$ separate atoms and on $\Gamma$. When each of the pairs of $\psi$ s is replaced by a particular term in the sum (12) for the propagator the delta distributions reduce the integral to one over matching connections on the $n$ atomic boundaries and

\footnotetext{
${ }^{11}$ Note also that the delta distributions in (12) are normalized Haar measure deltas. The definition (3) of $I_{0}$ is a Haar measure integral and the functional derivatives in (10) are Haar measure functional derivatives.
} 
$\Gamma$, where in each term the nodes are matched up according to the pairing of the $\psi \mathrm{s}$ and the boundary edges attached to each node are matched according to the permutation $\sigma \in S_{4}$ of the particular term of (12) being employed. (Actually, to make the matching of boundary edges corresponding to a given $\sigma$ unambigous we need to adopt a convention to specify the unpermuted order of these edges on the atoms and on $\Gamma$. Since boundary edges $e_{a}{ }^{b}$ are indexed by pairs of nodes $a, b$ the ordering of the $\psi \mathrm{s}$, which is equivalent to an ordering of the nodes, provides a natural choice for the ordering of the boundary edges attached to a particular node $a$.)

Glueing together the atoms and $\Gamma$ in this way produces an admissible 2-complex $J$ with boundary $\Gamma$. The corresponding contribution to (8) is

$$
\frac{\lambda^{n}}{n !}\left(\frac{1}{5 !}\right)^{n} \frac{1}{\operatorname{sym}(\Gamma)} \int d \mathbf{h} \theta^{*}\left(\mathbf{h}_{\Gamma}\right) w(\mathbf{h}) .
$$

To evaluate (8) we must add up the contributions (13) corresponding to each glueing, i.e. to each to each pairing of $\psi$ s with permutations $\sigma \in S_{4}$ associated to each pair. Since (13) depends only on the structure of $J$ we can first sum over glueings that lead to the same $J$ by multiplying (13) by the number of such glueings, and then sum over $J$ s.

In our expansion the atoms and the nodes in each atomic boundary as well as in $\Gamma$ are numbered, in an order arising from the way in which the (8) was written explicitly as an integral of a product of $\psi \mathrm{s}$. When the atoms and $\Gamma$ are glued together to form a 2-complex $J$ this reference numbering defines a numbering of the atoms, atomic boundary nodes and $\Gamma$ nodes of $J$. Conversely, if any admissible, $n$ atom 2 -complex bounded by $\Gamma$ is equiped with a "good" numbering then it defines uniquely a glueing of the atoms and $\Gamma$ with their reference numbering, and thus a pairing of the sequence of $\psi \mathrm{s}$ used in the expansion and a set of associated $S_{4}$ permutations. Here a good numbering is an arbitrary numbering of the atoms and of the nodes in each atom together with a numbering of the nodes of $\Gamma$ such that $\Gamma$ has the same adjacency matrix as in the reference numbering. From this we conclude, firstly, that our expansion generates all admissible, $n$ atom 2-complexes bounded by $\Gamma$, so the sum should run over all such complexes, and secondly, that all glueings that lead to the same 2-complex arise from good numberings of the atoms and nodes in that 2-complex.

There are $n !(5 !)^{n} \operatorname{sym}(\Gamma)$ good numberings, but renumbering does not necessarily produce a new glueing. A good renumbering of $J$ that produces 
the same gluing is a symmetry of $J$, because it defines a mapping of the set of cells of $J$ to itself that preserves all incidences, and conversely. There are thus $n !(5 !)^{n} \operatorname{sym}(\Gamma) / \operatorname{sym}(J)$ glueings that produce the complex $J$ so (8) equals

$$
\sum_{\left\{J \in \mathbf{A}_{\Gamma} \mid n(J)=n\right\}} \frac{\lambda^{n}}{\operatorname{sym}(J)} \int d \mathbf{h} \theta^{*}\left(\mathbf{h}_{\Gamma}\right) w(\mathbf{h}) .
$$

If the number of factors of $\psi$ in (8) is odd then $n$ atoms and $\Gamma$ together have an odd number of nodes and so cannot be matched up to form a 2-complex bounded by $\Gamma$. (14) therefore covers this case as well, because the integral (8) is zero while (14) is zero because $\left\{J \in \mathbf{A}_{\Gamma} \mid n(J)=n\right\}$ is empty. The theorem is thus established. I

Note that we could have restricted $\psi\left(h_{1}, \ldots, h_{4}\right)$ to be invariant under the gauge transformation

$$
h_{n} \mapsto g h_{n}
$$

without affecting the result. If one writes $\psi=\bar{\psi}+\Delta \psi$ where $\bar{\psi}=\int_{G} d g \psi(g \mathbf{h})$, the gauge average of $\psi$, then one finds $I_{0}[\psi]=I_{0}[\bar{\psi}]+I_{0}[\Delta \psi]$ while $\mathcal{V}[\psi]=$ $\mathcal{V}[\bar{\psi}]$ and $\Theta[\psi]=\Theta[\bar{\psi}]$ because $V$ and $\theta$ are gauge invariant. It follows that the integral over $\Delta \psi$ cancels between numerator and denominator in the expectation value

$$
\left\langle\Theta^{*}\right\rangle=\int \mathcal{D} \psi e^{-I_{0}[\psi]+\lambda \mathcal{V}[\psi]} \Theta^{*}[\psi] / \int \mathcal{D} \psi e^{-I_{0}[\psi]}
$$

and therefore this expectation value is unchanged if $\psi$ is replaced by $\bar{\psi}$, i.e. is restricted to functions invariant under (15). (In (6) the denominator of (16) was set to 1 . We may simultaneously set $\int \mathcal{D} \bar{\psi} e^{-I_{0}[\bar{\psi}]}=1$ and leave out the denominator also in the $\bar{\psi}$ formulation). In the perturbation series of the $\bar{\psi}$ field theory the propagator (12) is replaced by one that requires the connections to match only up to gauge. However, the gauge invariance of $V$ and $\theta$ imply that the integrals over the connection can be evaluated in a gauge in which the connections on the atomic boundaries and $\Gamma$ really do match. The integrals over the new gauge degrees of freedom at nodes then simply contribute factors of 1 because the normalized Haar measure is being used. 


\subsection{An example: The Turaev-Ooguri-Crane-Yetter model of BF theory}

A very simple gauge theory to which we can apply our scheme is BF theory. In this theory the action for the boundary value problem in which the connection is fixed on the boundary is $\int \operatorname{tr}[B \wedge F]$ where $F$ is the curvature of the connection, $B$ is a 2 -form which takes values in the Lie algebra of the gauge group $G$, and the trace is taken in this Lie algebra. Extremization of this action with respect to $B$ requires $F=0$, that is, a flat connection and in particular a flat connection on the boundary. Indeed a naive path integral quantization of this problem gives as the amplitude for the boundary connection a delta distribution with support on flat connections. It is therefore natural to discretize BF theory as a simplicial local lattice gauge theory in which the amplitude for the connection on the boundary of each simplex is a delta distribution with support on flat connections or, more concretely, $V$ is a delta distribution with support on flat connections on $\Gamma_{5}$. Here flatness on $\Gamma_{5}$ means that the holonomy around any closed loop of $\Gamma_{5}$ is trivial. The only such connections are the trivial connection and its gauge transforms, for which

$$
h_{i}{ }^{j}=p_{i} q^{i j}
$$

with $p_{i} \in G$ characterizing the gauge at the 4 -valent node $i$ while $q^{i j}=q^{j i} \in$ $G$ does so at the bivalent node in the chain connecting $i$ and $j$. Thus

$$
\begin{aligned}
V_{B F}\left(h_{i}{ }^{j}\left[h_{j}{ }^{i}\right]^{-1}\right) & =\prod_{k} \int d p_{k} \prod_{i<j} \delta\left(h_{i}{ }^{j}\left[h_{j}{ }^{i}\right]^{-1}, p_{i} p_{j}^{-1}\right) \\
& =\prod_{k} \int d p_{k} \prod_{l<m} \int d q^{l m} \prod_{i \neq j} \delta\left(h_{i}{ }^{j}, p_{i} q^{i j}\right) .
\end{aligned}
$$

Substituting $V_{B F}$ into the formula (田) for the interaction term in the field action we find

$$
\begin{aligned}
\mathcal{V}_{B F}[\psi] & =\frac{1}{5 !} \int_{G^{20}} d^{20} h V_{B F}\left(h_{i}{ }^{j}\left[h_{j}{ }^{i}\right]^{-1}\right) \psi\left(h_{1}{ }^{i}\right) \cdot \ldots \cdot \psi\left(h_{5}{ }^{i}\right) \\
& =\frac{1}{5 !} \prod_{k} \int d p_{k} \prod_{l<m} \int d q^{l m} \psi\left(p_{1} q^{1 j}\right) \cdot \ldots \cdot \psi\left(p_{5} q^{5 j}\right) \\
& =\frac{1}{5 !} \prod_{l<m} \int d q^{l m} \bar{\psi}\left(q^{1 j}\right) \cdot \ldots \cdot \bar{\psi}\left(q^{5 j}\right) .
\end{aligned}
$$


$I_{0}[\bar{\psi}]-\lambda \mathcal{V}_{B F}[\bar{\psi}]$ is precisely Ooguri's [17] action for generating BF theory summed over 2-complexes.

\section{Spin foam formulation}

\subsection{From local lattice gauge theories to spin foams}

The results of the previous section can also be understood within the spin foam framework. This has the advantage, among others, of replacing the functional integrals of (6) by more concrete multiple integrals over infinite sequences of real numbers, suggesting avenues toward regulating these integrals.

Any local lattice gauge theory with compact gauge group $G$ can be given a spin foam formulation. The spin foam formulation is conjugate to the connection formulation in the sense that if a connection is a history of the configuration variables of the model a spin foam is essentially a history of the momentum variables - the states in the spin foam picture are a sort of Fourier transform of the states in the connection picture.

The spin foam sum for the amplitude

$$
\int d \mathbf{h} \theta^{*}\left(\mathbf{h}_{\Gamma}\right) w(\mathbf{h}),
$$

of a boundary state $\theta$ on a fixed admissible 2-complex is obtained by expanding $V\left(\mathbf{h}_{x}\right)$ for each atom and $\theta\left(\mathbf{h}_{\Gamma}\right)$ on a basis of "spin network functions" [14], [4], and then integrating out the connection in each term of the resulting expansion of $\theta^{*} w$. Each non-zero term in this sum for (23) is associated to a spin foam. The translation of integrals over spacetime connections to sums over spin foams is described in detail in [6]. Here we only outline its main features. They key necessary element for any reformulation of this type is an orthonormal basis of the space of distributions on the gauge group manifold. Since $G$ is assumed to be compact the Peter-Weyl theorem assures us that the matrix elements of the unitary irreducible representations, ${ }^{[2} U^{(r)} m^{n}(g)$ (with $g$ ranging over $G, r$ labeling the representation, and $(m, n)$ the matrix

\footnotetext{
${ }^{12}$ Only one irreducible representation is selected from each class of unitarily equivalent representations. The basis of functions on $G$ consists of the matrix elements of these selected representations.
} 
elements) form an orthogonal basis of distributions. Indeed, since

$$
\int_{G} d g\left[U^{\left(r_{1}\right)}{ }_{m_{1}}^{n_{1}}(g)\right]^{*} U^{\left(r_{2}\right)}{ }_{m_{2}}^{n_{2}}(g)=\frac{1}{\operatorname{dim} r_{1}} \delta_{r_{1} r_{2}} \delta_{m_{2}}^{m_{1}} \delta_{n_{1}}^{n_{2}}
$$

with $\operatorname{dim} r$ the dimensionality of the representation $r$, the set $\left\{\sqrt{\operatorname{dim} r} U^{(r)}{ }_{m}^{n}\right\}_{r, m, n}$ is an orthonormal basis.

Any function of the parallel propagators along the edges of a graph may thus be expanded in terms of tensor products of representation matrices of the parallel propagators. Notice that a representation matrix of the parallel transporter along an edge is a two point tensor, with one index living at each end of the edge. The subspace of functions that are gauge invariant at a given vertex is thus spanned by functions obtained from the tensor product of representation matrices by contracting the indices at that vertex with an invariant tensor, also called an "intertwiner". (An example of an intertwiner when $G=S U(2)$ is the antisymmetric tensor $\epsilon_{i j k}$, which corresponds to a trivalent vertex with each incident edge carrying the spin 1 representation). An orthonormal basis of fully gauge invariant functions can be found by first selecting at each vertex an orthonormal basis $\left\{W_{I}^{\mathbf{r}}\right\}_{I}$ of the space of intertwiners for each set $\mathbf{r}$ of incident representations that admits intertwiners ${ }^{\text {[t] }}$ and then forming the basis functions by contracting factors $\sqrt{\operatorname{dim} r_{e}} U^{\left(r_{e}\right)}{ }_{m}^{n}\left(g_{e}\right)$ for the edges $e$ with intertwiners belonging to the chosen intertwiner bases at the nodes. The data labeling a basis function, namely the representation on each edge and the basis intertwiner at each node, define a "spin network" on the graph,

Now consider expanding $V(\mathbf{g})$ into a sum of spin network basis functions.

$$
V(\mathbf{g})=\sum_{\mathbf{r}_{. .}, \mathbf{I} .} A^{\left(\mathbf{r}_{.} .\right)} \mathbf{I} \cdot\left[\prod_{i<j} \sqrt{\operatorname{dim} r_{i j}}\right] \phi_{\mathbf{I} . .}^{(\mathbf{r} .)}(\mathbf{g}) .
$$

(Recall $\left.g_{i j}=h_{i}{ }^{j}\left[h_{j}{ }^{i}\right]^{-1}\right) . \phi_{\mathbf{I}}^{\left(\mathbf{r}_{\text {.. }}\right)}(\mathbf{g})$ is the (normalized) basis spin network function on $\Gamma_{5}$ with intertwiners $W_{I_{i}}$ at the 4 -valent nodes and representations

\footnotetext{
13 That is to say, each $\mathbf{r}=\left[r_{1}, r_{2}, \ldots, r_{v}\right]$ such that $r_{1} \otimes \ldots \otimes r_{v}$ contains the trivial representation, so that non-zero invariant tensors exist.

${ }^{14}$ Strictly the spin network consists of the subgraph carrying carrying non-trivial representations - edges that carry trivial representations are left off - together with the "colouring" data consisting of the non-trivial representations and the intertwiners. In our present context of lattice gauge theory this point is of no importance but in the continuum in which there is no convenient graph of all possible edges it is essential.
} 
$r_{i j}$ on the edges connecting the 4-valent nodes.

$$
\phi_{\mathbf{I} . .}^{(\mathbf{r} .)}(\mathbf{g})=\prod_{i<j} \sqrt{\operatorname{dim} r_{i j}} U^{\left(r_{i j}\right)}{ }_{m_{i j}}^{n_{i j}}\left(g_{i j}\right) \prod_{k} W_{I_{k} n_{1 k} \ldots n_{k-1 k}} m_{k k+1 \cdots m_{k 5}} .
$$

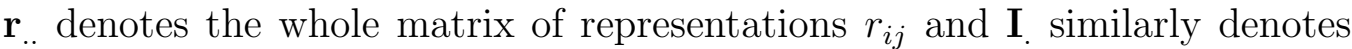
the sequence of basis intertwiners $I_{i}$. The factor $\left[\prod_{i<j} \sqrt{\operatorname{dim} r_{i j}}\right]$ in (25) has been separated from the coefficient $A^{(\mathbf{r} . .)} \mathbf{I}$. for later convenience.

Each spin network on the boundary of an atom defines a spin foam on the body of the atom: The intertwiner $I_{i}$ on the 4 -valent node $i$ is the spin foam intertwiner on the spoke connecting the node with the 0-cell at the center of the atom; The representation $r_{i j}$ on the chain connecting nodes $i$ and $j$ defines the representation on the wedge of the atom bounded by the chain.

The orthogonality relation (24) and the orthogonality of the basis intertwiners then ensure that when $\theta^{*} w$ is integrated over the connection the only terms that remain are those in which these atomic spin foams match up, in the sense that the intertwiners on the two halves of a 1-cell of $J$ agree and the representations on all the wedges of a 2-cell agree.". Moreover, they ensure that the intertwiners and representations match at the boundary $\Gamma$ where the spin foam meets the spin network in the expansion of $\theta$.

The weight, or amplitude, of each spin foam $S$ in the sum for (23) that remains once the connection is integrated out is

$$
c_{\partial S}^{*} \prod_{x \in 0 \text {-cells of } J} A^{(\mathbf{r} . .) \mathbf{I} .} \prod_{y \in 2 \text {-cells of } J} \operatorname{dim} r_{y}
$$

\footnotetext{
15 They must agree in the sense that if two wedges of a 2-cell have matching ("coherent") orientations then they must carry the same representation, whereas if they have opposite orientations they must have complex conjugate representations. These representations will be discussed more a little further on.

16 This formula should be viewed as somewhat schematic some details have been ignored. The matching of basis intertwiners on coincident nodes is not between basis intertwiners in the same invariant tensor space but rather in dual spaces. If we use a raised index $I$ to label the dual basis intertwiners we see that the matching of intertwiner indices $A$ in the product (27) should always be between a downstairs and an upstairs index. For many groups one may choose the intertwiner bases so that raising or lowering the intertwiner index is trivial, leaving $A$ unchanged, but we are not sure that this is always true. Sign factors, not included in our formula, are also present for "psuedo-real" representations that some groups have which depend on the relative orientations of the coincident boundary edges on which neighboring atoms meet. The proper resolution of these issues is explained in outline at the end of this section.
} 
where $c_{\partial S}$ proportional to the coefficient of the spin network $\partial S$ (the boundary of the spin foam) in the expansion of $\theta$, being obtained from the coefficient by dividing by a factor of $\sqrt{\operatorname{dim} r}$ for each edge of $\Gamma$.

\subsection{Divergences, regularization, and renormalization}

Spin foam models can have divergences when $J$ has closed 2-surfaces. For instance, in the model of BF theory that we have discussed the weight $w$ of a connection has redundant delta distributions, and is thus infinite, whenever $J$ contains topological 2-spheres. Other closed surfaces can also contribute singular factors ${ }^{\top 7}$

In the spin foam sum the infinities take the form of a divergence of the sum over representations and can thus be regulated by somehow cutting off this sum. This can be accomplished by replacing $G$ with the quantum group $G_{q}$ with $q \neq 1$ a root of unity, which has a finite set of inequivalent irreducible representations. The spin foams are coloured with the representations and intertwiners of $G_{q}$, and $A$ is replaced by its natural generalization to such representations 16 18 yielding a finite sum. One can also simply cut off the sum, leaving the model otherwise unchanged.

In lattice models of topological theories, or Yang-Mills theories for that matter, the continuum limit theory is usually defined not by a sum over lattices but by the limiting values of the observables of the theory as the lattice is refined. In the case of simplicial models the existence of this limit requires that the model be independent of the triangulation used (at least so long as the triangulation is very fine, i.e. has very many simplices). This requires that the regulated model be renormalized, because the number of divergences, and thus the number of the large, regulator dependent factors in the transition amplitudes, depends on the simplicial complex used.

17 The singular factor associated with a closed surface in BF theory is

$$
\sum_{r \in R}(\operatorname{dim} r)^{\chi}+\sum_{r \in P R}(-\operatorname{dim} r)^{\chi}+\kappa \sum_{r \in C}(\operatorname{dim} r)^{\chi}
$$

where $\chi$ is the Euler characteristic of the surface, $\kappa=1$ if the surface is orientable and 0 if it is not, $R$ is the set of (unitary equivalence classes of) real irreducible representations, $P R$ is the set of psuedo-real irreps, and $C$ is the set of complex irreps (for definitions see 35 ). When $G=U(1)$ we obtain a singular factor for every orientable closed surface, whereas for $G=S U(2)$ only the four surfaces with Euler characteristic $\chi \geq 0$ create divergences. 
For BF theory on a triangulated manifold this means dividing out a factor $\delta(0)_{\text {regulated }}=\left[\sum_{r}(\operatorname{dim} r)^{2}\right]_{\text {regulated }}$ for each independent 2-sphere in $J$. This is the origin of the factor $\delta(0)_{\text {regulated }}^{N_{0}-N_{1}}$ (with $N_{0}$ the number of 0-simplices and $N_{1}$ the number of 1-simplices in the triangulation) in the weight of a history in simplicial BF theory [18]. Renormalization is more subtle in the context of a general admissible 2-complex because the spheres do not necessarily span all the closed 2-surfaces (for instance one can make a 2-complex having a torus as it's only closed surface). As far as we know the renormalization of the model has not been carried out in this wider context.

In the present paper we are of course advocating a different approach to the continuum limit, in which one sums over 2-complex spacetimes. We have not studied the issue of renormalization in this approach.

\subsection{The field theory in terms of spin foams}

Now let's see how the field theory (2) can be expressed in terms of spin foams and networks. While this is qualitatively quite straightforward it turns out to be a little tricky in detail. The difficulty has to do with the fact that the spin network basis is not quite unique. Some conventions have to be introduced to define a particular basis. When translating from an integral over connections to a sum over spin foams on a particular, given, 2-complex one can choose the spin network bases on the atomic boundaries so as to simplify the calculations on that particular 2-complex. On the other hand, in order to express our field theory in spin foam terms, which boils down to expressing it in terms of the coefficients in spin network expansions of $V, \theta$, and $\psi$, we have to choose a particular spin network basis on the prototypical atomic boundary $\Gamma_{5}$. This is then the basis on every atomic boundary in the complexes generated by the field theory. The resulting inflexibility in the choice of basis complicates the calculation of the amplitudes of Feynman diagrams.

We have taken a compromise route. We present the field theory in spin foam language using a basis that makes the presentation as simple and sy-

\footnotetext{
18 The expansion on the basis of matrix elements of the Haar measure delta distribution on $G$ is$$
\delta(g)=\sum_{r} \operatorname{dim} r \operatorname{tr} U^{(r)} .
$$ 
metrical as possible, obtaining a very neat result. Then we outline how the amplitudes of the Feynman diagrams can be calculated by exploiting the fact that one is free to change to adapted spin network bases independently for each Feynman diagram, making the calculation as simple as in a spin foam model on a fixed 2-complex spacetime.

It may well be that the results could be presented more cleanly using a slightly more abstract "basis independent" approach to the spin network basis, in which the conventional choices that fix a particular basis are not made. Here we will stick with definite bases.

Let us begin the translation of the field theory by expanding $\psi$ using the Peter-Weyl theorem:

$$
\psi(\mathbf{h})=\sum_{\mathbf{r}} b^{(\mathbf{r}) \mathbf{m}}{ }_{\mathbf{n}} \prod_{t=1}^{4} \sqrt{\operatorname{dim} r_{t}} U^{\left(r_{t}\right)}{ }_{m_{t}}^{n_{t}}\left(h_{t}\right) .
$$

The symmetry of $\psi$ under permutations of its arguments requires the coefficients $b$ to be similarly symmetric:

$$
b^{(\mathbf{r}) \mathbf{m}_{\mathbf{n}}}=b^{(\sigma[\mathbf{r}]) \sigma[\mathbf{m}]}{ }_{\sigma[\mathbf{n}]} \forall \sigma \in S_{4} .
$$

The free action is easily expressed in terms of these coefficients if we write it as $I_{0}[\psi]=\frac{1}{2 \cdot 4 !} \int_{G^{4}} \psi^{*} \psi d^{4} h$. This is valid because $\psi$ is real. We find, using (24), that

$$
I_{0}[\psi]=\frac{1}{2 \cdot 4 !} \sum_{\mathbf{r}}\left[b^{(\mathbf{r}) \mathbf{m}}\right]^{*} b^{(\mathbf{r}) \mathbf{m}}{ }_{\mathbf{n}}
$$

The corresponding propagator is

$$
\left\langle b^{\left(\mathbf{r}_{1}\right) \mathbf{m}_{1}}{ }_{\mathbf{n}_{1}}\left[b^{\left(\mathbf{r}_{2}\right) \mathbf{m}_{2}}{ }_{\mathbf{n}_{2}}\right]^{*}\right\rangle_{0}=\sum_{\sigma \in S_{4}} \delta_{\mathbf{r}_{1} \sigma\left[\mathbf{r}_{2}\right]} \delta_{\sigma\left[\mathbf{m}_{2}\right]}^{\mathbf{m}_{1}} \delta_{\mathbf{n}_{1}}^{\sigma\left[\mathbf{n}_{2}\right]}
$$

with $\delta_{\mathbf{a}}^{\mathbf{b}}=\prod_{t=1}^{4} \delta_{a_{t}}^{b_{t}}$.

To express the interaction term $\mathcal{V}$ in terms of the coefficients $b$ in as clean a way as possible we shall introduce a further representation theoretic tool. If $g_{12} \in G$ defines parallel transport on the edge from node 1 to node 2 then $g_{21}=\left[g_{12}\right]^{-1}$ defines parallel transport along the inverse edge from 2 to 1 . A

\footnotetext{
${ }^{19}$ It can be evaluated by translating the field propagator $\left\langle\psi(\mathbf{h}) \psi\left(\mathbf{h}^{\prime}\right)\right\rangle_{0}$ or directly from (32). In carrying out the Gaussian integral in the second approach the reality conditions satisfied by $b$ (spelled out further on in the text) must be taken into account.
} 
unitary representation matrix $U_{m_{1}}^{(r) m_{2}}\left(g_{12}\right)$ can be expressed in terms of $g_{21}$ as $\left[U^{(r)}\left(g_{21}\right)\right]_{m_{1}}^{-1 m_{2}}=\left[U_{m_{2}}^{(r) m_{1}}\left(g_{21}\right)\right]^{*}$. It is just the parallel propagator from 2 to 1 in the complex conjugate of the representation $r$. Thus a spin network function of the parallel transporters along the oriented edges of a graph can be expressed just as well in terms of parallel transporters of the edges with different orientations, provided the representations on the reversed edges are replaced by their complex conjugates.

Now recall that in a spin network basis the representations $U^{(r)}$ that may be placed on edges are particular representatives chosen one out of each unitary equivalence class. Moreover, in order to keep the definition of the spin network basis simple we shall use the same set of representatives on all edges. In general the conjugate representation $\left[U^{(r)}\right]^{*}$ will not be the representative $U^{\left(r^{*}\right)}$ of its own equivalence class, it will only be unitarily equivalent to it. For instance, all representations of $S U(2)$ are equivalent to their conjugates, but unitary irreducible representations of non-integer spin are not real and so are not equal to their conjugates.

We therefore introduce $\varepsilon_{m n}^{(r)}$, a unitary matrix such that

$$
\left[U^{(r)}\right]^{*}=\varepsilon^{(r) \dagger} U^{\left(r^{*}\right)} \varepsilon^{(r)}
$$

and let $\varepsilon^{(r) m n}=\left[\varepsilon_{m n}^{(r)}\right]^{*}$, which is the inverse of $\varepsilon_{m n}^{(r)}$ in the sense that $\varepsilon_{m x}^{(r)} \varepsilon^{(r) n x}=$ $\delta_{m}^{n}$. A few notes on $\varepsilon^{(r)}$ :

- It follows from (34) that $\varepsilon_{m n}^{(r)}$ is an invariant tensor (an intertwiner) with $m$ a covector index of the representation $r^{*}$ and $n$ a covector index of the representation $r$.

- (34) determines $\varepsilon^{(r)}$ up to phase. Consequently $\varepsilon^{\left(r^{*}\right) m n}$ equals $\varepsilon^{(r) n m}$ up to a phase factor, and thus when $r=r^{*}, \varepsilon^{(r)}$ is either purely symmetric or antisymmetric. ${ }^{20}$ When $r \neq r^{*}$ one is of course free to choose the representative of the equivalence class of the conjugate representation as one likes. One could simply choose the conjugate representation itself and use $\varepsilon_{m n}^{(r)}=\delta_{m n}$. We are therefore free to set $\varepsilon^{(r)}=\varepsilon^{\left(r^{*}\right)}$ for all $r$. $^{2 r}$ • $\varepsilon^{(r)}$ can be used as a

${ }^{20}$ For example consider the spin $1 / 2$ and spin 1 representations of $S U(2)$ in the standard eigenbasis of the generator $J_{z}$. In the spin $1 / 2$ representation $\varepsilon_{m n}^{\left(\frac{1}{2}\right)}=\epsilon_{m n}$, while in the spin 1 representation $\varepsilon_{m n}^{(1)}=\delta_{m,-n}$, so it is antisymmetric for spin $1 / 2$ and symmetric for spin 1 . Indeed one finds that $\varepsilon_{m n}^{(j)}$ is symmetric for all integer $j$ and antisymmetric for all half odd integer $j$.

${ }^{21}$ When $r=r^{*}$ and $\varepsilon^{(r)}$ is symmetric the representation $r$ is real; If $r=r^{*}$ and $\varepsilon^{(r)}$ is antisymmetric $r$ is psuedo-real; Finally, if $r \neq r * r$ is complex. See [35]. 
(possibly antisymmetric) metric to turn a representation $r$ vector (carrying an upstairs index) into a representation $r^{*}$ covector (carrying a downstairs index) according $a_{m}^{\left(r^{*}\right)}=\varepsilon_{m x}^{(r)} a^{(r) x}$. The inverse, index raising, operation is $a^{(r) x}=\varepsilon^{(r) x m} a_{x}^{\left(r^{*}\right)}$. Of course complex conjugation also turns unitarily transforming $r$ vectors into $r^{*}$ covectors and vice versa. The index positioning in all our equations is consistent with this fact.

The relation (34) can be used to define a modified, more symmetric spin network expansion of $V$ which depends only minimally on the orientations chosen for the edges of $\Gamma_{5}$. Notice that

$$
\begin{aligned}
U_{m_{1}}^{(r) n}\left(h_{1} h_{2}^{-1}\right) & =U_{m_{1}}^{(r) x}\left(h_{1}\right)\left[U_{n}^{(r) x}\left(h_{2}\right)\right]^{*} \\
& =U_{m_{1}}^{(r) x}\left(h_{1}\right) \varepsilon_{x y}^{(r)} U_{m_{2}}^{\left(r^{*}\right) y}\left(h_{2}\right) \varepsilon^{(r) n m_{2}} .
\end{aligned}
$$

Thus adopting the notation $r_{i j}=r_{j i}^{*}$ for $i>j$ we may write

$$
V=\sum_{\mathbf{r} ., \mathbf{I} .} A^{\left(\mathbf{r}_{. .}\right) \mathbf{I}} \cdot\left[\prod_{i \neq j} \sqrt{\operatorname{dim} r_{i j}}\right] U^{\left(r_{i j}\right)}{ }_{m_{i j}}^{x_{i j}}\left(h_{i}{ }^{j}\right) \prod_{k} W_{I_{k}}^{m_{k 1} \ldots m_{k k-1} m_{k k+1} \ldots m_{k 5}} \prod_{i<j} \varepsilon_{x_{i j} x_{j i}}^{\left(r_{i j}\right)} .
$$

The lower indices of the intertwiners appearing in (25) have been raised using $\varepsilon$. The invariance of $\varepsilon$ implies that the resulting tensors $W_{I_{k}}^{\mathbf{m}_{k}}$ are also invariant and thus intertwiners for the incident representations $r_{k i}$. It is easy to verify that they form an orthonormal basis of such intertwiners. (37) is thus a spin network expansion of $V$ with the bivalent nodes assigned the intertwiners $\varepsilon_{x_{i j} x_{j i}}^{\left(r_{i j}\right)}$ (which is $\sqrt{\operatorname{dim} r_{i j}}$ times the unique (mod phase) normalized bivalent intertwiner). In (37) the orientations of the edges manifest themselves only in the ordering of the indices in these $\varepsilon$ s.

Evaluating $\mathcal{V}$ is now straightforward. If we use the reality of $\psi$ to replace $\psi$ by $\psi^{*}$ in (4) the integrals can be carried out using (24) and we obtain

$$
\left.\mathcal{V}=\frac{1}{5 !} \sum_{\mathbf{r}_{. .}, \mathbf{I} .} A^{\left(\mathbf{r}_{. .}\right) \mathbf{I} \cdot} \prod_{k}\left[b^{\left(\mathbf{r}_{k} .\right) \mathbf{m}_{k .} .}\right]_{k}\right]^{*} W_{I_{k}}^{\mathbf{m}_{k} .} \prod_{i<j} \varepsilon_{x_{i j} x_{j i}}^{\left(r_{i j}\right)} .
$$

$\mathcal{V}$ is even simpler when expressed in terms of the spin network coefficients in an expansion of the "gauge invariant" field $\bar{\psi}(\mathbf{h})=\int_{G} \psi(g \mathbf{h}) d g$. A spin network type expansion of this field can be obtained by inserting into the expansion (30) of $\psi$ the projector

$$
\int_{G} \prod_{t=1}^{4} U^{\left(r_{t}\right)}{ }_{m_{t}}^{n_{t}}(g) d g=\sum_{I}\left[W_{I}^{(\mathbf{r}) m_{1} m_{2} m_{3} m_{4}}\right]^{*} W_{I}^{(\mathbf{r}) n_{1} n_{2} n_{3} n_{4}}
$$


onto invariant $r_{1} \otimes r_{2} \otimes r_{3} \otimes r_{4}$ tensors. Thus

$$
\bar{\psi}(\mathbf{h})=\sum_{\mathbf{r}, I} c^{(\mathbf{r}) I}{ }_{\mathbf{n}} W_{I}^{(\mathbf{r}) \mathbf{m}} \prod_{t=1}^{4} \sqrt{\operatorname{dim} r_{t}} U^{\left(r_{t}\right)}{ }_{m_{t}}{ }^{n_{t}}\left(h_{t}\right)
$$

with $c^{(\mathbf{r}) I}{ }_{\mathbf{n}}=b^{(\mathbf{r}) \mathbf{m}} \mathbf{n}_{\mathbf{n}}\left[W_{I}^{(\mathbf{r}) \mathbf{m}}\right]^{*}$. In terms of the coefficients $c$ the interaction term is

$$
\mathcal{V}=\frac{1}{5 !} \sum_{\mathbf{r}_{. .}, \mathbf{I} .} A^{\left(\mathbf{r}_{. .}\right) \mathbf{I}} \prod_{k}\left[c^{\left(\mathbf{r}_{k} .\right) I_{k}} \mathbf{x}_{k}\right]^{*} \prod_{i<j} \varepsilon_{x_{i j} x_{j i}}^{\left(r_{i j}\right)},
$$

It's just $A$ times the result of contracting together the indices of five $c^{*}$ in the pattern of a 4-simplex. More precisely it can be obtained from (37) by replacing the intertwiners $W_{I}$ by $\left[c^{I}\right]^{*}$ and setting all the parallel transporters to 1 . This prescription can also be applied to $\theta$ to express $\Theta$ in terms of the coefficiants $c^{*}$.

$\varepsilon$ also allows us to express the reality conditions satisfied by the coefficients $b: \psi^{*}=\psi$ and (34) implies that

$$
b^{\left(\mathbf{r}^{*}\right) \mathbf{m}} \mathbf{n}=\left[b^{(\mathbf{r}) \mathbf{x}} \mathbf{y}\right]^{*} \varepsilon^{(\mathbf{r}) \mathbf{m} \mathbf{x}} \varepsilon_{\mathbf{n y}}^{(\mathbf{r})}
$$

$\left(\right.$ with $\left.\varepsilon^{(\mathbf{r}) \mathbf{m n}}=\prod_{t=1}^{4} \varepsilon^{\left(r_{t}\right) m_{t} n_{t}}\right)$.

We are now in a position to rewrite the functional integral (6) as an ordinary integral over the coefficients $b$ (which are finite in number if the spin foam model has been regulated by cutting off the sum over representations). Moreover we may evaluate the Feynman diagrams of the perturbation series in $\lambda$ using the propagator (33) and the reality conditions (42).

What we have given is just about the simplest, most symmetric statement of the spin foam formulation of the theory. However, it is not the most convenient form for actually evaluating the Feynman diagrams. In fact it is best to first select the Feynman diagram one wishes to evaluate, corresponding to a particular 2-complex $J$, and then choose the spin network basis for expanding $\Theta^{*}$ and the factor of $\mathcal{V}$ corresponding to each atom. To define this adapted set of spin network bases we choose an orientation for each 1-cell and 2 -cell of $J$. The orientations on the 2-cells induce an orientation on each edge of $\Gamma$ and each atomic boundary edge that matches the sense of circulation of the 2-cell that it cuts. The orientations on the 1-cells define a sign on each node, which is positive if the 1-cell is outgoing at the node and negative if it is incoming. Now we choose spin network basis functions corresponding to 
these orientations of the edges. We obtain an expansion of $V$, rather like that given in (25) with coherent orientations on the chain of two edges connecting a pair of nodes. However, we do not necessarily have two outgoing and two incoming edges at each node as in (25). The intertwiner bases are also adapted. At each positive node a basis $\left\{W_{I}^{(\mathbf{r})}\right\}$ with indices suitably lowered for incoming edges, is used. At the corresponding negative node (the negative node that lies on the same 1-cell) the intertwiners $\left[W_{I}^{(\sigma[\mathbf{r}])}\right]^{*}$ are used. Here $\sigma \in S_{4}$ is the mapping of the incident edges at the negative node to the corresponding incident edges at the positive node. These complex conjugate and permuted intertwiners are still orthonormal and have the index positions compatible with our convention for the orientations of the edges.

With these conventions (and with the correspondingly adjusted coefficients $A$ and $c_{\partial S}$ ) the amplitude of the Feynman diagram is relatively easy to obtain. The result is as described in (27): It is a sum over histories consisting of representations and basis intertwiners, with only histories in which every 2-cell $y$ carries a single representation $r_{y}$ - the common representation on the atomic boundary edges that cut the 2-cell and on the edges of $\Gamma$ that bound it - and each 1-cell carries a single intertwiner basis index $I$, the common value of the indices at the positive and negative nodes on the 1-cell. Such a history is a spin foam. The amplitude for each history is the product of the coefficients $A$ for the atoms times $c_{\partial S}^{*}$ and a factor $d i m r_{y}$ for each 2-cell. This last factor results from the factors of $\varepsilon_{x_{i j} x_{j i}}^{\left(r_{i j}\right)}$ that appear in (38) and (41), associated to each wedge. When the orientations of the edges are changed to match those of the 2-cells these are replaced by Kronecker deltas. Then, when the propagators for $b$ are substituted in, the Kronecker deltas associated to wedges in a given 2-cell end up being contracted in a chain around around the 2-cell, thus contributing a factor $\operatorname{dim} r_{y}$.

\section{Some closing remarks}

\subsection{On the possible finiteness of the field theory cor- responding to regulated spin foam models}

The number of admissible 2-complexes increases very rapidly with the number of atoms. In fact the number of complexes of $n$ atoms with a given 
boundary $\Gamma$ having $m$ 4-valent nodes is given approximately by

$$
\sum_{\left\{J \in g o t h A_{\Gamma} \mid n(J)=n\right\}}=\left(\frac{4 !}{2}\right)^{(5 n+m) / 2} \frac{(5 n+m) !}{([5 n+m] / 2) !} \frac{1}{n !(5 !)^{n}} \frac{1}{\operatorname{sym}(\Gamma)} .
$$

This increases as $(n !)^{3 / 2}$ for large $n$, so unless the vertex function $V$ is very special indeed the radius of convergence of the series (17) is zero.

What prospect is there of making sense of the functional integral (6)? In fact it is not unreasonable to hope that once the spin foam model is regulated by cutting off the sums over representations (as we discussed in the context of BF theory) that the integral (6) can be assigned a finite value by analytic continuation. We will suppose that the finite set of representations summed over in the cut off model includes the representation $r^{*}$ whenever it includes $r$. Then $\psi$ in the cut off model is a real function on $G^{4}$ determined by a finite set of parameters. These paramenters can be the $b^{(\mathbf{r}) \mathbf{m}}{ }_{\mathbf{n}}$, but we will use a set of linear combinations $\left\{x_{p}\right\}_{p=1}^{N}$ of these chosen so that they are real and $I_{0}[\psi]=\mathbf{x}^{T} \mathbf{x}$. Then (6) becomes

$$
\int_{\mathbf{R}^{N}} d^{N} x e^{-\mathbf{x}^{T} \mathbf{x}} e^{\lambda \mathcal{V}^{p_{1} \ldots p_{5}} x_{p_{1}} \cdots \cdot x_{p_{5}}} \Theta^{* q_{1} \ldots q_{m}} x_{p_{1}} \cdot \ldots \cdot x_{p_{m}},
$$

a function of $\lambda$ which we shall call $F(\lambda)$. For simplicity we will suppose that $V(\mathbf{g})$ is real, as is the case in BF theory, and in the models of [6] and [27], then also $\mathcal{V}^{n_{1} \ldots n_{5}}$ is real and we see that the integral is convergent when $\lambda$ is pure imaginary - the integral is a Gaussian times a function of modulus $O\left(|x|^{m}\right)$. Can the result be analytically continued away from the imaginary axis?

To see that the answer is quite possibly "yes" consider the simplest analog of the integral (44):

$$
f(\lambda)=\int_{-\infty}^{\infty} d x e^{-x^{2}} e^{\lambda x^{5}}
$$

The coefficients in the formal power series expansion of $f$ about $\lambda=0$ also diverge as $(n !)^{3 / 2}$ for large $n$ and, like (44) the integral converges for purely imaginary $\lambda$. Moreover, $f$ can be continued to the entire complex plane, with a fivefold branch cut extending from 0 to $\infty$. To show this one first writes $f$ as $f(\lambda)=h(\lambda)+h(-\lambda)$ where

$$
h(\lambda)=\int_{0}^{\infty} d x e^{-x^{2}} e^{\lambda x^{5}}
$$


This integral is convergent when $\Re \lambda \leq 0$. It may be reexpressed in terms of $\lambda^{-1 / 5}$ and the rescaled integration variable $y=\lambda^{1 / 5} x$. From this form one obtains a series for $h$ in powers of $\lambda^{-1 / 5}$ that is convergent for all finite values of this parameter, and the claimed result follows.

The first step in this argument can be repeated for the regulated functional integral (44). Let $M$ be the union of rays in $\mathbf{R}^{4}$ on which $\mathcal{V}^{n_{1} \ldots n_{5}} x_{n_{1}}$. $\ldots \cdot x_{n_{5}} \leq 0$. Then

$$
F(\lambda)=H(\lambda)+H(-\lambda)
$$

with

$$
H(\lambda)=\int_{M} d^{N} x e^{-\mathbf{x}^{T} \mathbf{x}} e^{\lambda \mathcal{V}(\mathbf{x})} \Theta^{*}(\mathbf{x}) .
$$

Again we find that the integral converges for $\Re \lambda \leq 0$. The question is now whether it can be continued beyond this domain. It seems likely that this would be the case for generic models, especially as it is finite on $\Re \lambda=0$. If it is continuable then $F(\lambda)$ will be defined and finite on an open region in the complex plane, possibly including $\lambda=1$ which is the value corresponding most closely to a simple sum of the spin foam model over all admissible 2-complexes.

\subsection{Generalizations of the formalism}

Our formalism can easily be generalized to a wider class of 2-complexes. We have allowed only atoms which are dual 2-skeletons of 4-simplices, which means that they have five spokes each of which is four valent. The field theory can be extended so that it generates 2-complexes including atoms with any given number $p$ of 4 -valent spokes (which are dual to 4-polyhedra bounded by $p 3$-simplices). All that is necessary is to include a suitable interaction term in the action (2) formed from a vertex function for the new type of atom like $\mathcal{V}$ was formed from the vertex function $V$ and $\Theta$ from the boundary state $\theta$. The expression (23) for the amplitude of the state $\theta$ on the boundary $\Gamma$ of $J$ can be viewed as the partition function for a closed 2-complex consisting of $J$ and one copy of a new type of atom with boundary $-\Gamma$ ( $\Gamma$ with reversed orientation) and vertex function $\theta^{*}$. Adding $\Theta^{*}$ to the action would generate all complexes consisting of both this new type of atom and the original type.

Even with the above generalization all atoms have spokes of valence four and thus all bounding graphs of atoms and of complexes have non-trivial nodes of valence four only. The theory may be generalized to acomodate other 
valences $q$ by introducing additional fields depending on $q$ group elements instead of four like $\psi$ does, and adding a quadratic free action to the total action for each such field.

Any model having a finite set of types of atoms can be handled by these means, including of course models with spacetimes of any dimensionality to the extent that this dimensionality can be captured in the combinatorial structure of the atoms.

\subsection{Matrix models}

When our formalism is applied to two dimensional models with atoms dual to triangles we find that the analog of $c^{(\mathbf{r}) I}{ }_{\mathbf{n}}$ is $c^{\left(r_{1}\right)}{ }_{n_{1} n_{2}} \equiv b^{\left(r_{1}, r_{2}\right) m_{1} m_{2}}{ }_{n_{1} n_{2}} \frac{1}{\sqrt{d i m r_{1}}} \delta_{r_{1} r_{2}^{*}} \varepsilon_{m_{1} m_{2}}^{\left(r_{1}\right)}$ $\left(r_{1}\right.$ has to equal $r_{2}^{*}$ because $r_{1} \otimes r_{2}$ contains the trivial representation only in this case. $\varepsilon^{(r)} / \sqrt{\operatorname{dim} r}$ is the only normalized bivalent intertwiner). $c^{(r)}$ is essentially the matrix of matrix models [34]: The triangular matrix model is recovered by choosing the vertex function to be $V\left(g_{i j}\right)=A(\operatorname{dim} r)^{3} / 3 ! \operatorname{tr} U^{(r)}\left(g_{12} g_{23} g_{31}\right)$ so that only one representation $r$ appears in its spin network expansion. Then $\mathcal{V}=A / 3 ! c_{n_{1}}^{(r) n_{2}} c_{n_{2}}^{(r) n_{3}} c_{n_{3}}^{(r) n_{1}}$

\section{Acknowledgements}

We thank Roberto DePietri for discussions and help, and M.R. would like to thank Rodolfo Gambini for a discussion on the subject of this paper. This work was partially supported by NSF grant PHY-95-15506, PPARC grant PPA/6/s/1998/00613 and a gift from the Jesse Phillips Foundation.

\section{References}

[1] R De Pietri, L Freidel, K Krasnov, C Rovelli, "Barrett-Crane model from a Boulatov-Ooguri field theory over a homogeneous space", Nuclear Physics, to appear, hep-the/9907154

[2] M Reisenberger, C Rovelli, "Spin foams as Feynman diagrams", grqc/0002083. 
[3] J. Iwasaki. A definition of the Ponzano-Regge quantum gravity model in terms of surfaces. J. Math. Phys., 36:6288, 1995. gr-qc 9410010.

[4] M. Reisenberger. Worldsheet formulations of gauge theories and gravity. talk given at the 7th Marcel Grossmann Meeting Stanford, July 1994. gr-qc 9412035, 1994.

[5] M. Reisenberger and C. Rovelli. "Sum over surfaces" form of Loop Quantum Gravity. Phys. Rev. D, 56:3490, 1997.

[6] M. Reisenberger. A lattice worldsheet sum for 4-d Euclidean general relativity. gr-qc/9711052.

[7] J. Baez. Class. Quant. Grav, 15(1998) 1827-1858; "An Introduction to Spin Foam Models of Quantum Gravity and BF Theory" gr-qc/9905087; "Spin Foam Perturbation Theory" gr-qc/9910050.

[8] C Rovelli, "The projector on physical states in loop quantum gravity", gr-qc/9806121.

[9] R. De Pietri, Canonical "loop" quantum gravity and spin foam models, to appear in the proceedings of the XXIIIth Congress of the Italian Society for General Relativity and Gravitational Physics (SIGRAV), 1998, grqc/9903076.

[10] R. Gambini and A. Trias. Gauge dynamics in the C-representation. Nucl. Phys. B, 278:486, 1986; R. Gambini and J. Pullin. Loops, Knots and Quantum Gravity. Cambridge University Press (1997).

[11] C Rovelli, L Smolin Rhys. Rev. Lett. 61, 1155, (1988); Nucl. Phys. B331 (1), 80-152, (1990). C Rovelli "Loop Quantum Gravity", Living Reviews in Relativity, electronic journal, http://www.livingreviews.org/Articles/Volume1/1998-1rovelli; also in gr-qc/9709008. M Gaul, C Rovelli, "Loop quantum gravity and the meaning of diffeomorphism invariance" in Towards Quantum Gravity. Proceedings of the XXXV Karpacz International Winter School on Theoretical Physics J Kowalski-Glikman ed (Springer Verlag, Heidelberg, 1999). 
[12] A. Ashtekar, J. Lewandowski, D. Marolf, J. Mourão, and T. Thiemann. Quantization of diffeomorphism invariant theories of connections with local degrees of freedom. Journ. Math. Phys., 36:519, 1995

[13] J. A. Zapata. A combinatorial approach to diffeomorphism invariant quantum gauge theories. J. Math. Phys. 38 (1997) 5663-5681. grqc/9703037; J. A. Zapata. Combinatorial space from loop quantum gravity. Gen. Rel. Grav.30 (1998) 1229-1245. gr-qc/9703038.

[14] C Rovelli and L Smolin, "Spin Networks and Quantum Gravity", Phys Rev D52 (1995) 5743, gr-qc/9505006. J. Baez, Adv. Math. 117 (1996) 253.

[15] G. Ponzano and T. Regge. Semiclassical limit of Racah coefficients. In F. Bloch, editor, Spectroscopic and group theoretical methods in physics, page 1, Amsterdam, 1968. North Holland.

[16] V Turaev, "Quantum invariants of 3-manifolds and a glimpse of shadow topology" in Quantum Groups, Springer Lecture Notes in Mathematics 1510, pp 363-366 (Springer-Verlag, New York, 1992); Quantum Invariants of Knots and 3-Manifolds (de Gruyter, New York, 1994).

[17] H. Ooguri. Topological lattice models in four dimensions. Mod. Phys. Lett. A, 7:2799, 1992.

[18] L. Crane and D. Yetter, "A Categorical construction of 4-D topological quantum field theories", in Quantum Topology, L. Kaufmann and R. Baadhio eds., World Scientific, Singapore 1993. hep-th/9301062. L. Crane, L. Kauffman and D. Yetter, J. Knot Theor. Ramifications 6 (1997) 177-234, hep-th/9409167.

[19] C Rovelli: "Basis of the Ponzano-Regge-Turaev-Viro-Ooguri quantum gravity model is the loop representation basis", Phys Rev D48 (1993) 2702. TJ Foxon, "Spin networks, Turaev-Viro theory and the loop representation", Class Quant Grav 12 (1995) 951-964, gr-qc/9408013.

[20] A. Ashtekar, C. Rovelli, and L. Smolin. Weaving a classical geometry with quantum threads. Phys. Rev. Lett., 69:237, 1992 
[21] C. Rovelli and L. Smolin. Discreteness of volume and area in quantum gravity. Nucl. Phys. B, 442:593, 1995. Erratum:Nucl. Phys. B, 456:734, 1995.

[22] R. DePietri and C. Rovelli. Geometry eigenvalues and scalar product from recoupling theory in loop quantum gravity. Phys. Rev. D, 54:2664, 1996; S. Fritelli, L. Lehner, and C. Rovelli. The complete spectrum of the area from recoupling theory in loop quantum gravity. Class. Quantum Grav., 13:2921, 1996.

[23] A. Ashtekar and J. Lewandowski. Quantum theory of geometry I: area operators. Class. Quant. Grav.14 (1997), A55-A82, gr-qc 9602040.

[24] T. Thiemann. A length operator for canonical quantum gravity. $J$. Math. Phys. 39 (1998) 3372-3392. gr-qc/9606099.

[25] L. Freidel. 3D quantum Lorentzian geometry. CGPG Seminar, Penn State University, April 1999.

[26] M. Reisenberger. A left-handed simplicial action for Euclidean general relativity. Class. Quantum Grav., 14:1753, 1997. gr-qc/9609002

[27] J. W. Barrett and L. Crane, J. Math. Phys. 39 (1998) 3296.

[28] J. Iwasaki. A surface theoretic model of quantum gravity. gr-qc/9903119.

[29] F. Markopolou and L. Smolin. Causal evolution of spin networks. Nucl. Phys. B508 (1997). gr-qc/9702025; F. Markopolou and L. Smolin. Quantum geometry with intrinsic local causality. Phys. Rev. D58 (1998). gr-qc/9702025

[30] S. Gupta. Causality in spin foam models. gr-qc/9908018

[31] J. W. Barrett and L. Crane. A Lorentzian signature model for quantum general relativity. $g r-q c / 9904025$

[32] G. Horowitz, Comm. Math. Phys. 125 (1989) 417. M. Blau and G. Thompson, Phys. Lett. B228 (1989) 64.

[33] D. V. Boulatov, Mod. Phys. Lett. A7 (1992) 1629. 
[34] F. David, Nucl. Phys. B257 (1985) 45. J. Ambjorn, B. Durhuus, J. Frolich, Nucl. Phys. B257 (1985) 433. V. A. Kazakov, I. K. Kostov, A. A. Migdal, Phys. Lett. 157 (1985) 295. D. V. Boulatov, V. A. Kazakov, I. K. Kostov, A. A. Migdal, Nucl. Phys. B275 (1986) 641. M. Douglas, S. Shenker, Nucl. Phys. B335(1990) 635. D. Gross, A. Migdal, Phys. Rev. Lett. 64 (1990) 635; E. Brezin, V. A. Kazakov, Phys. Lett. B236 (1990) 144.

[35] H. Georgi Lie Algebras in Particle Physics: From Isospin to Unified Theories, Benjamin/Cummings, 1982. 